\title{
Potential Penetration of CTAB- and MUDA-coated Gold Nanorods into Tooth Enamel
}

\author{
So Ran Kwon ${ }^{1}$, Yiming Li ${ }^{2}$, Elvin M Walemba ${ }^{3}$, Krassimir N Bozhilov ${ }^{4}$, Christopher Perry ${ }^{5}$
}

\begin{abstract}
Aim: Gold nanorods (GNRs) have gained interest as a promising carrier for antibiotics. Gold nanorods may reduce the development of antimicrobial resistance in certain microbial species. Although applications of GNRs to mitigate oral biofilms are under development, their use in the oral cavity may have adverse effects. The aim of this study was to evaluate the potential penetration of GNRs into the tooth enamel structure using confocal laser scanning microscopy (CLSM) and scanning transmission electron microscopy (STEM).

Materials and methods: Our approach was to synthesize GNRs with cationic [cetyltrimethylammoniumbromide (CTAB)] and anionic [11-mercaptoundecanoic acid (MUDA)] surface coatings. We hypothesized that penetration would be surface coating-dependent.

Results: Regardless of the chemical modification of the GNRs of size $\sim 20 \mathrm{~nm} \times 8 \mathrm{~nm}$, exposure of these materials did not result in superficial penetration into the enamel.

Conclusion: Within the limitations of this study, it is concluded that the use of CLSM and STEM is a feasible approach to investigate the penetration of nanomaterials into the tooth structure.

Clinical significance: Exposure of the enamel with chemically modified GNRs of size $\sim 20 \mathrm{~nm} \times 8 \mathrm{~nm}$ will not result in superficial penetration into the enamel.

Keywords: Confocal laser scanning microscopy, Enamel, Gold nanorods, Penetration, Scanning transmission electron microscopy.

The Journal of Contemporary Dental Practice (2020): 10.5005/jp-journals-10024-2834
\end{abstract}

\section{INTRODUCTION}

The concept of nanotechnology, which is the ability to control and manipulate atoms and molecules, was introduced by physicist Richard Feynman in $1959 .^{1,2}$ Since then, nanotechnology has revolutionized the scope and depth of medical and dental applications. In dentistry, nanotechnology has enabled a shift from the mechanistic restoration of tooth repair to an emphasis on diagnosis and disease-prevention mechanisms. ${ }^{3}$

In the era of nanotechnology, bold ideas have been suggested such as formulation of microdental robots that would be programd to survey all teeth surfaces and break down harmful materials, or changing the superficial enamel layer with materials like sapphire or diamond to enhance the toughness and appearance of teeth. ${ }^{4}$ Moreover, there is a time progression for these technologies to transition from laboratory bench to dental applications. This is to ensure safety aspects and timely identification of weaknesses and strengths, which are critical when it comes to introducing a new material into the clinical setting. ${ }^{3}$

Gold nanomaterials have received widespread interest because of their easy preparation with controlled size, ready conjugation with biomolecules, and highly tunable optical properties. Thus, by varying the shape of the nanogold material, optical and thermal properties can be controlled. This has found applications in photodynamic or theranostic applications where heat can be generated to cure disease by varying the photon energy. ${ }^{5}$ Functional gold nanorods (GNRs) have been used as an alternative delivery vehicle or as a potent antimicrobial agent against multidrug-resistant bacteria. ${ }^{6}$ Therefore, the use of functional GNRs is expected to drive the development of groundbreaking formulations to prevent and treat biofilm-related dental diseases. This is achieved by GNRs affecting the oral biofilm via theranostic and/or alternative delivery vehicle approaches.

\footnotetext{
${ }^{1,2}$ Center for Dental Research, Loma Linda University School of Dentistry, Loma Linda, California, USA

${ }^{3}$ Faculty of Science, Asia-Pacific International University, Loma Linda, California, USA

${ }^{4}$ Central Facility for Advanced Microscopy and Micro, University of California at Riverside, California, USA

${ }^{5}$ Division of Biochemistry, Loma Linda University School of Medicine, Loma Linda, California, USA
}

Corresponding Author: So Ran Kwon, Center for Dental Research, Loma Linda University School of Dentistry, Loma Linda, California, USA, Phone: +1 909558 5118, e-mail: sorankwon@llu.edu

How to cite this article: Kwon SR, Li Y, Walemba EM, et al. Potential Penetration of CTAB- and MUDA-coated Gold Nanorods into Tooth Enamel. J Contemp Dent Pract 2020;21(5):475-480.

Source of support: Colgate Award for Research Excellence Grant (\#2160391).

Conflict of interest: None

The development of biofilm on the tooth surface is a complex process that occurs over a 2- to 3-day period. The process begins with adsorption of a sticky film onto the tooth surface derived from bacterial and host molecules and continues with biofilm maturation and biofilm dispersal. ${ }^{7}$ Once formed, it has been recognized as a virulence factor in many oral infectious diseases, including dental caries, periodontitis, and endodontic infections. ${ }^{8}$ Identification of innovative antimicrobial strategies to control the oral biofilm has been a central issue over the past years. However, the effect of new therapeutic agents and the potential penetration of these agents into the tooth have not been investigated. The human enamel is well known to act as a semipermeable membrane to allow for continuous dynamic ion exchange with the oral biofilm, with calcium 
phosphate apatite crystals moving in both directions to maintain a proper mineral balance. ${ }^{9}$ Recent evidence indicates that some organic material in the enamel originates from exogenous sources and becomes part of the organic matrix. ${ }^{10}$ The enamel prisms though compactly organized and structured have pore sizes of approximately $2-6 \mathrm{~nm}$ that would make this interchange feasible. ${ }^{11}$

The use of nanomaterials in the oral cavity is promising but may cause certain effects on the tooth enamel that are not documented. The purpose of this study was to evaluate potential penetration of GNRs into the tooth enamel structure. The aim was to synthesize GNRs with cationic [cetyltrimethylammoniumbromide (CTAB)] and anionic [11-mercaptoundecanoic acid (MUDA)] surface coatings. We characterized the formation of cationic and anionic GNRs and evaluated the penetration of differently charged GNRs into extracted human teeth. We hypothesized that accumulation and penetration into the enamel would be dependent on the type of chemical modification of GNRs as determined with confocal laser scanning microscopy (CLSM) and scanning transmission electron microscopy (STEM) imaging.

\section{Materials and Methods}

The study was performed at Loma Linda University School of Dentistry. The use of extracted human teeth with no identifiers was approved by the Loma Linda University Institutional Review Board (IRB).

\section{Synthesis of CTAB-coated Gold Nanorods (3-5 Aspect Ratio)}

The ammonium hydroxide (28-30\%), $\mathrm{NaOH}(\geq 98 \%)$, silver nitrate ( $\geq 99 \%)$, gold (III) chloride hydrate $\left(\mathrm{HAuCl}_{4} \cdot \times \mathrm{H}_{2} \mathrm{O} ; 99.999 \%\right.$ trace metals basis), ascorbic acid (reagent grade), and sodium borohydride $(<90 \%)$ were used as received (Sigma-Aldrich, Milwaukee, WI, USA). Single-crystal GNRs of aspect ratio 3:5 were synthesized as per published methods. ${ }^{12}$ In the GNR synthesis, the solutions were maintained at $27-30^{\circ} \mathrm{C}$ using a water bath. Gold nanoparticle seeds were first synthesized, and then added to the CTAB growth solution. To make the seeds, $25 \mu \mathrm{L}$ of $50 \mathrm{mM} \mathrm{HAuCl}_{4}$ solution was added to $4.7 \mathrm{~mL}$ of $0.1 \mathrm{M} \mathrm{CTAB}$ solution and the mixture thoroughly mixed and left in the water bath for 5 minutes. Then, $300 \mu \mathrm{L}$ of a freshly prepared $10 \mathrm{mM} \mathrm{NaBH}_{4}$ ( $7.6 \mathrm{mg} \mathrm{NaBH}_{4}$ in $20 \mathrm{~mL}$ water under ice) solution was rapidly injected into the CTAB solution, while it was being vortexed. After 10-20 seconds, the seed solution was stirred mildly (400 rpm). For the growth solution, $\mathrm{HAuCl}_{4}(100 \mu \mathrm{L}, 50 \mathrm{mM})$ was added to CTAB $(10 \mathrm{~mL}, 100 \mathrm{mM})$; the mixture was gently shaken and kept for 10 minutes in a water bath to ensure complexation between gold salt and CTAB. Afterward, ascorbic acid solution (75 $\mu \mathrm{L}, 100 \mathrm{mM}$ ) was added to the mixture, which was gently shaken for few seconds (the solution turns colorless). A solution of $\mathrm{AgNO}_{3}$ $(80 \mu \mathrm{L}, 5 \mathrm{mM})$ was added to the growth solution and shaken for few seconds. Finally, the seed solution $(120 \mu \mathrm{L})$ was added to the mixture and the solution vigorously shaken for the last time and then left undisturbed at $27^{\circ} \mathrm{C}$ for 30 minutes in a water bath.

\section{Chemical Modification to MUDA-coated Gold Nanorods}

The chemical classes of materials were modeled by chemical modification of the GNRs $(\sim 2-5 \mathrm{~nm})$ using alkyl thiols. The traditional approach is via the thiol functionality on the alkyl chain (RSH), which binds chemically to gold to form thiolate
Au-S-R. We adopted this approach with GNRs to chemically modify CTAB coating with 11-mercaptoundecanoic acid. The method of diafiltration was used to purify the CTAB-coated GNRs. ${ }^{13}$ After washing, the samples were left at room temperature overnight to precipitate out the excess CTAB crystals (which are only soluble above room temperature) and the supernatant decanted. Then, $5 \mathrm{~mL}$ of the GNR supernatant was added to a glass vial and mixed with $5 \mathrm{~mL}$ of MUDA solution, which is comprised of $4 \mathrm{~mL}$ of water (pH adjusted to 9.0) and $1 \mathrm{~mL}$ of $10 \mathrm{mM}$ of MUDA in ethanol. The mixture was heated with stirring in a water bath to about $80^{\circ} \mathrm{C}$ for 10 minutes to allow efficient ligand replacement of CTAB with MUDA. After cooling at room temperature, the solution was transferred to microcentrifuge tubes and sonicated for 1 hour. The MUDA GNR solutions were then centrifuged twice at 10,000 rpm for 20 minutes and suspended in $10 \mathrm{mM}$ Tris buffer.

\section{Characterization of Gold Nanorods}

Both CTAB-coated and MUDA-coated GNRs were characterized by atomic force (AFM) and electron microscopies as well as UV-vis and dynamic light scattering (DLS). Au NRs solutions were dried on silanated mica discs and examined by atomic force microscopy with a Multimode 8 scanning probe microscope (Bruker, Santa Barbara, CA, USA) in the peak force tapping mode and using ScanAsystTM ( $k=0.4 \mathrm{~N} \mathrm{~m}^{-1}, f=70 \mathrm{kHz}$ ) air probes. Samples were prepared for electron microscopy measurements by centrifuging $(10,000 \times g)$ twice to remove excess reagents and suspended in water followed by dropping 5-10 $\mu \mathrm{L}$ of solution onto a carboncoated Cu grid (Ted Pella 200 mesh) and allowing the samples to air dry. UV-vis spectra were recorded using a Varian Cary 300 spectrophotometer equipped with a temperature controller. All UV-vis measurements were made using a quartz cell $(1 \mathrm{~cm}$ path length, $25^{\circ} \mathrm{C}$ ) unless otherwise indicated. Particle sizes were determined using a Nicomp Z3000 Zeta Potential/Particle Sizer (PSS Nicomp, USA) equipped with a He-Ne laser wavelength of $\lambda=638$ $\mathrm{nm}$ and a power output of $60 \mathrm{~mW}$. All data were collected at $25^{\circ} \mathrm{C}$ and at a scattering angle of $90^{\circ}$ with a square acrylic cuvette $(3 \mathrm{~mL}$ volume) containing a suspension of diffusible particles. Values of the refractive index, $n=1.33$, and viscosity, $\eta=8.9 \times 10^{-4} \mathrm{Ns} \mathrm{m}^{-2}$, for water were assumed to be applicable to the solutions. Prior to measurements, all samples were centrifuged ( $8,000 \times g, 30$ minutes) to remove the excess surfactant and suspended in deionized water. Zeta potential measurements (average of three replicates taken at 1-minute intervals) were done using $20 \mu \mathrm{L}$ sample and diluting in $2 \mathrm{~mL} 10^{-2} \mathrm{M} \mathrm{NaCl}$ solution.

\section{Treatment of Human Enamel Slabs}

Extracted sound human third molars $(N=18)$ were collected and stored in $0.2 \%$ sodium azide solution at $4^{\circ} \mathrm{C}$. Teeth were cleaned of gross debris, sectioned, and the crown portion cut to a rectangular shape of $4 \times 6 \times 4 \mathrm{~mm}^{3}$. The sectioned specimens were then embedded in acrylic resin to expose the enamel surface only. The enamel specimens were immersed in $500 \mu \mathrm{L}$ of $0.1 \mathrm{M}$ Tris-buffer (Control, $N=6$ ), $500 \mu \mathrm{L}$ of $0.1 \mathrm{M}$ CTAB-coated GNRs (CTAB, $N=$ 6), and $500 \mu \mathrm{L}$ of $0.1 \mathrm{M}$ MUDA-coated GNRs (MUDA, $N=6$ ) for 3 weeks at $37^{\circ} \mathrm{C}$. The solutions were replenished every week. The concentration of the GNRs was determined by the method of Orendorff and Murphy. ${ }^{14}$ Half of the specimens from each group were used for confocal laser scanning microscopy and the other half of each group was used for scanning transmission electron microscopy imaging. 


\section{Confocal Laser Scanning Microscopy}

On completion of 3 weeks' immersion in respective solutions, specimens were rinsed with Milli-Q water and immersed in 100 $\mu \mathrm{M}$ Rhodamine $\mathrm{B}$ solution for 24 hours to counterstain the dental hard tissue. The images of gold nanorod penetration into enamel were acquired in a laser scanning confocal microscope (Zeiss LSM 710 NLO, Jena, Germany). A 750 nm fs Ti:Sapphire laser (Coherent Chameleon Vision II Ti) was used as the excitation source for the gold nanorod imaging while the dental hard tissue was imaged with a laser at $561 \mathrm{~nm}$. An oil immersion objective (Plan-Apochromat $63 \times / 1.40$ Oil DIC M27) and a matched pinhole were used in all experiments. With the z-scan function, 3D images were obtained in ZEN computational software to evaluate the penetration depths of the GNRs into the enamel tissue.

\section{STEM Sample Preparation}

On completion of 3 weeks' immersion in respective solutions, STEM lamellae were prepared from a polished cross-section of the tooth specimen following established procedures with a dualbeam scanning electron microscope and FIB instrument (Quanta 200i 3D, ThermoFisher Scientific). First, a strap of 5 - $\mu$ m-thick carbon layer was deposited over a region of interest on the polished cross-section using the ion beam ( $30 \mathrm{kV}, 0.5 \mathrm{nA})$. Two trenches were then milled out $(30 \mathrm{kV}, 10 \mathrm{nA})$ on either side of a $15-\mu \mathrm{m}$-thick slice of material. The slice of material was reduced to $1 \mu \mathrm{m}$ thickness ( $30 \mathrm{kV}, 5 \mathrm{nA}$ ). The slice was then cut free from the substrate on three sides, leaving only a small connecting bridge. An in situ tungsten nanomanipulator probe (Autoprobe200, Oxford Instruments Inc.) was attached to the free side of the substrate by depositing $\mathrm{Pt}(2 \mathrm{kV}, 8 \mathrm{nA})$. The remaining connection to the substrate was milled away $(30 \mathrm{kV}, 0.5 \mathrm{nA})$ and the probe was retracted with the sample. The sample was then welded to a copper TEM half-grid (Omniprobe) using Pt deposition and the connection to the probe was milled away (30 kV, $0.5 \mathrm{nA})$.
The lamella was thinned to $500 \mathrm{~nm}$ at $30 \mathrm{kV}, 1 \mathrm{nA}$ on both sides at $\pm 4^{\circ}$ incidence angle grazing milling condition. To reduce surface amorphization and gallium implantation, final milling at $5 \mathrm{kV}$ and $0.5 \mathrm{nA}$ was used to thin the sample to approximately $80 \mathrm{~nm}$.

\section{STEM Imaging and Analysis}

STEM imaging was performed at $300 \mathrm{kV}$ accelerating voltage in a ThermoFisher Scientific Titan Themis 300 instrument, fitted with X-FEG electron source, 3 lens condenser system, and S-Twin objective lens. STEM images were recorded with Fischione Instruments Inc. Model 3000 High angle annular dark field (HAADF) Detector with probe current of $150 \mathrm{pA}$, frame size of 2,048 $\times 2,048$, dwell time of $15 \mu \mathrm{sec} / \mathrm{pixel}$, and camera length of $245 \mathrm{~mm}$.

Energy-dispersive X-ray spectroscopy (EDS) analyzes and elemental mapping were obtained in the STEM at $300 \mathrm{kV}$, utilizing the ThermoFisher Scientific SuperX system equipped with $4 \times$ $30 \mathrm{~mm}^{2}$ window-less SDD detectors symmetrically surrounding the specimen with a total collection angle of 0.68 srad, by scanning the thin-foil specimens. Elemental mapping was performed with an electron beam probe current of $350 \mathrm{pA}$ at 1,024 × 1,024 frame resolution. EDS spectra were extracted from the mapped areas and processed using the Bruker Instruments Esprit 1.9 software. Quantification is based on the Cliff-Lorimer approach using calculated $\mathrm{k}$-factors and correcting for absorption by estimating foil thickness.

\section{RESULTS}

\section{Synthesis and Characterization of Gold Nanorods}

Figure 1 illustrates the methodology used in this study, from GNRs synthesis to application on the tooth enamel surface. Figure 2 shows the normalized UV-vis spectra of the CTAB- and MUDAcoated GNRs suspended in $10^{-2}$ Tris buffer. Two plasmon peaks are observed at $\sim 524$ (transverse) and $\sim 744 \mathrm{~nm}$ (longitudinal) in the

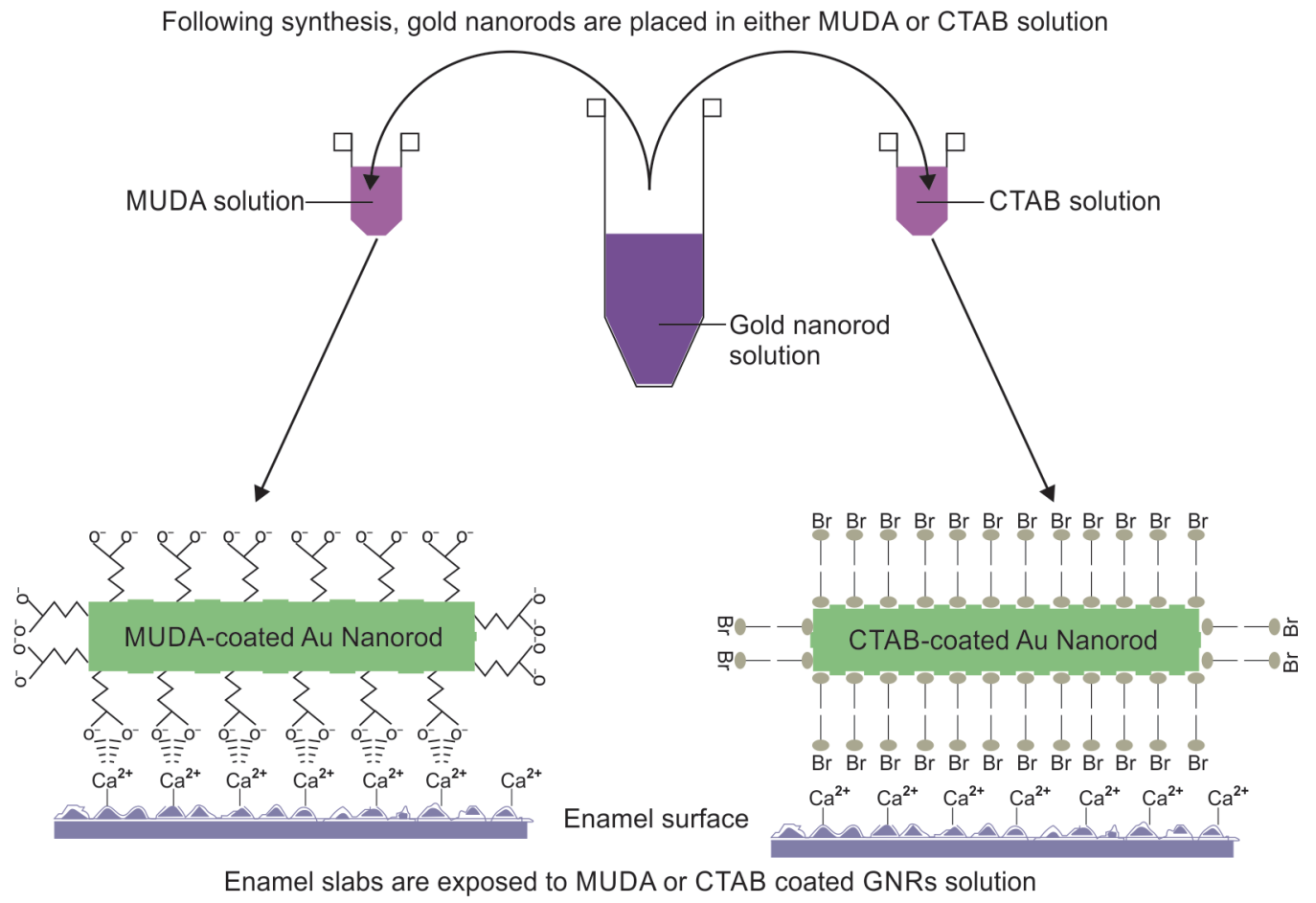

Fig. 1: Graphical illustration of study design 
UV-vis. The longitudinal plasmon peak position is dependent on the aspect ratio of the NR; for nanorods with higher-aspect ratios, the longitudinal plasmon peaks will red-shift to longer wavelength. Chemical modification by the MUDA red-shifted the longitudinal plasmon peak from $\sim 744$ to $756 \mathrm{~nm}$. This is because the refractive index around the GNR interface is changed due to the MUDA coating. The aspect ratio and morphology were determined by STEM. The size of GNRs was consistent and determined to be $\sim 20$ $\mathrm{nm} \times 8 \mathrm{~nm}$ (Fig. 3). Average zeta potential measurements of -1.50 and $-28.74 \mathrm{mV}$ were recorded for initial CTAB- and MUDA-modified GNRs, respectively. The significantly negative zeta potential for MUDA-coated GNRs is from the deprotonated carboxylic acid groups, where the $\mathrm{pH}$ is above $\mathrm{p} K_{\mathrm{a}}$ of MUDA (5.5).

\section{Confocal Laser Scanning Microscopy}

Figure 4 show the 3-D image of the human enamel stained with Rhodamine B to contrast the images of MUDA- and CTAB-coated

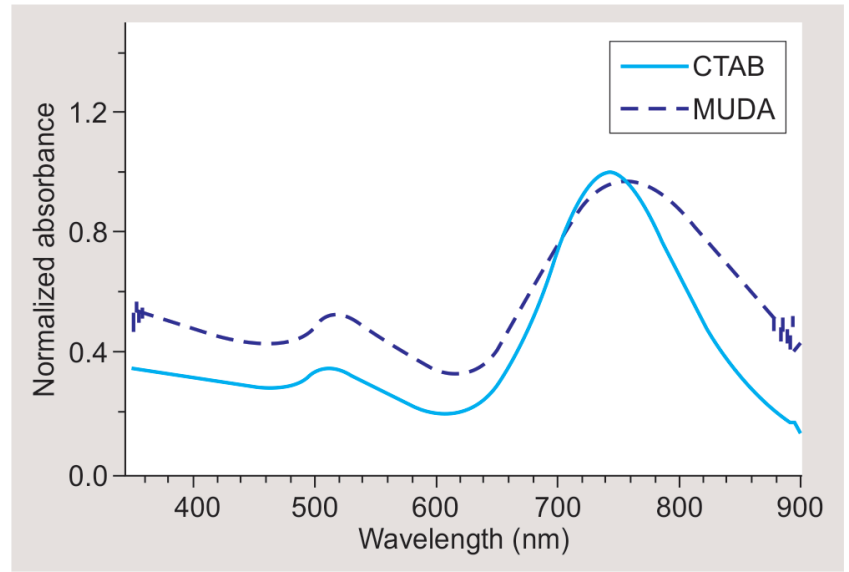

Fig. 2: UV-vis spectrum of cetyltrimethylammoniumbromide and 11-mercaptoundecanoic acid coated gold nanorods
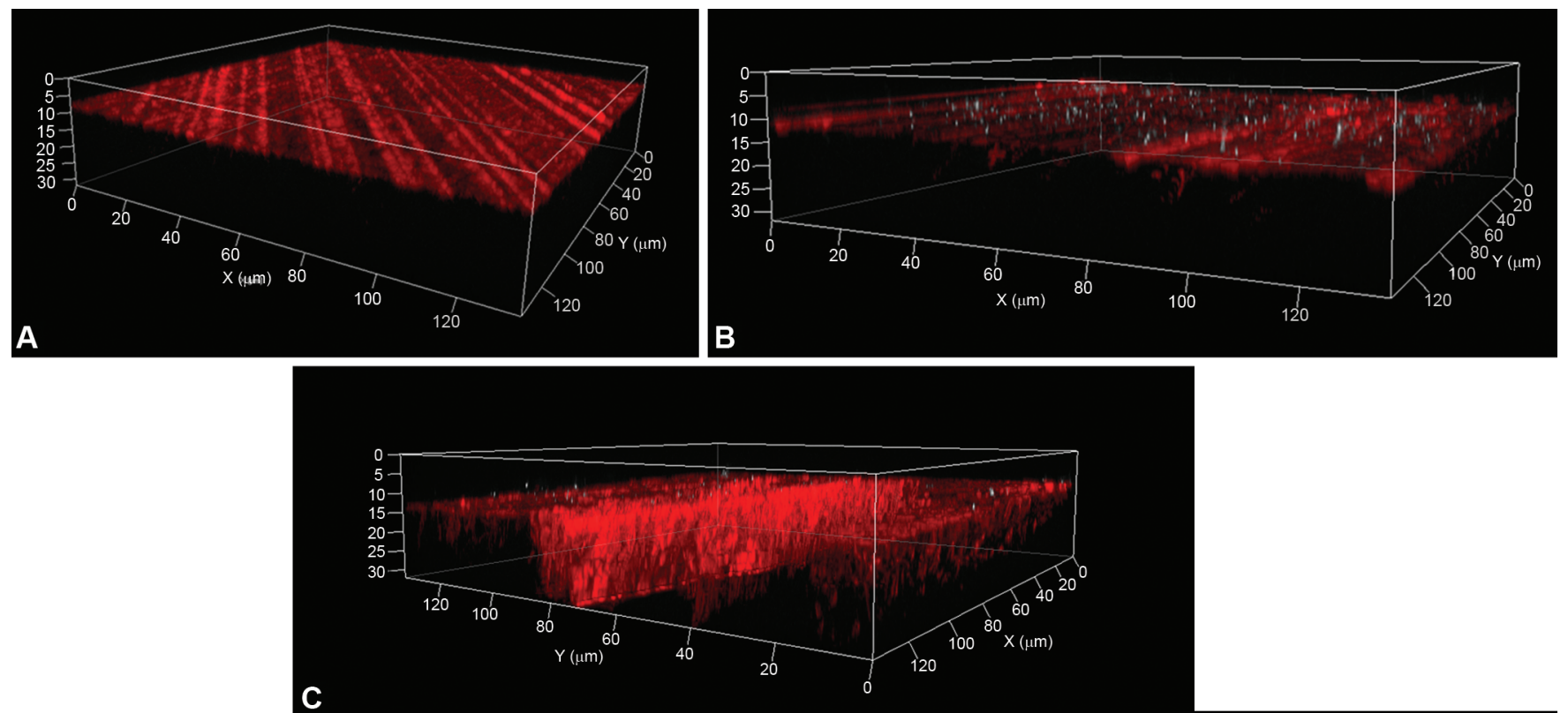

Figs 4A to C: Three dimensional confocal images of teeth exposed to Tris buffer or gold nanorods; (A) Tris buffer solution; (B) Cetyltrimethylammoniumbromide-coated gold nanorods solution; (C) 11-Mercaptoundecanoic acid-coated gold nanorods solution

GNRs. The enamel is primarily composed of hydroxyapatites in the rod and interrod enamel. In the enamel, Rhodamine B penetrated along the interprismatic spaces so that the longitudinal orientation of the rods was clearly visible. Surface striations formed by the polishing process were visible on all enamel samples. Accumulation of scattered MUDA- and CTAB-coated GNRs was limited to the outer surface while the control group showed a clean enamel surface.

\section{STEM Imaging and Analysis}

STEM samples of $\approx 80 \mathrm{~nm}$ thickness were prepared from a polished cross-section of the tooth specimen with a dual-beam scanning electron microscope and focus ion beam instrument. Additionally, the EDS analysis was obtained in STEM (Fig. 5). The micrographs in Figure 5 represent enamel specimens treated with Tris buffer the negative control where no GNRs were visible (Fig. 5) and detectable with EDS (Fig. 5). Immersion of enamel specimens in CTAB GNRs solution showed localized accumulation of GNRs onto the surface,

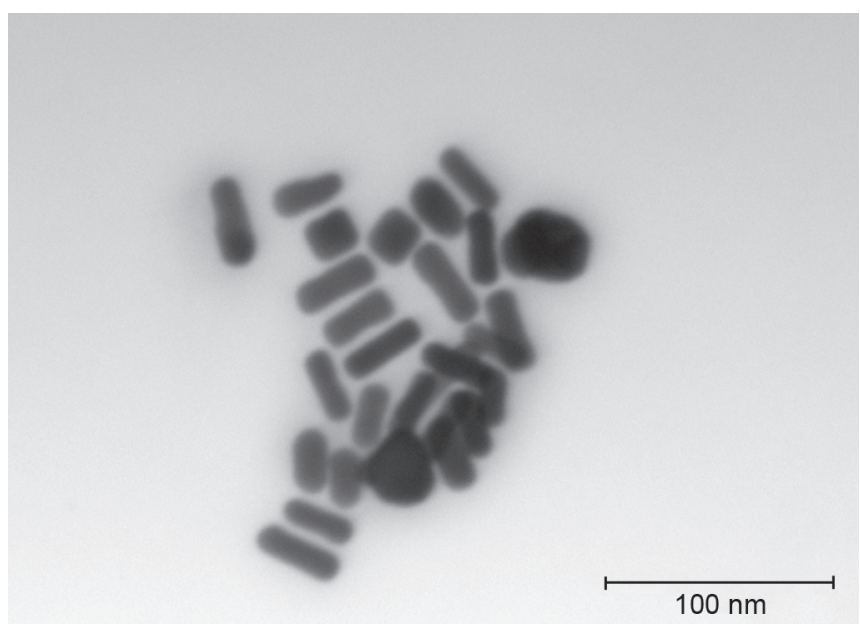

Fig. 3: Scanning transmission electron microscopy image of gold nanorods 

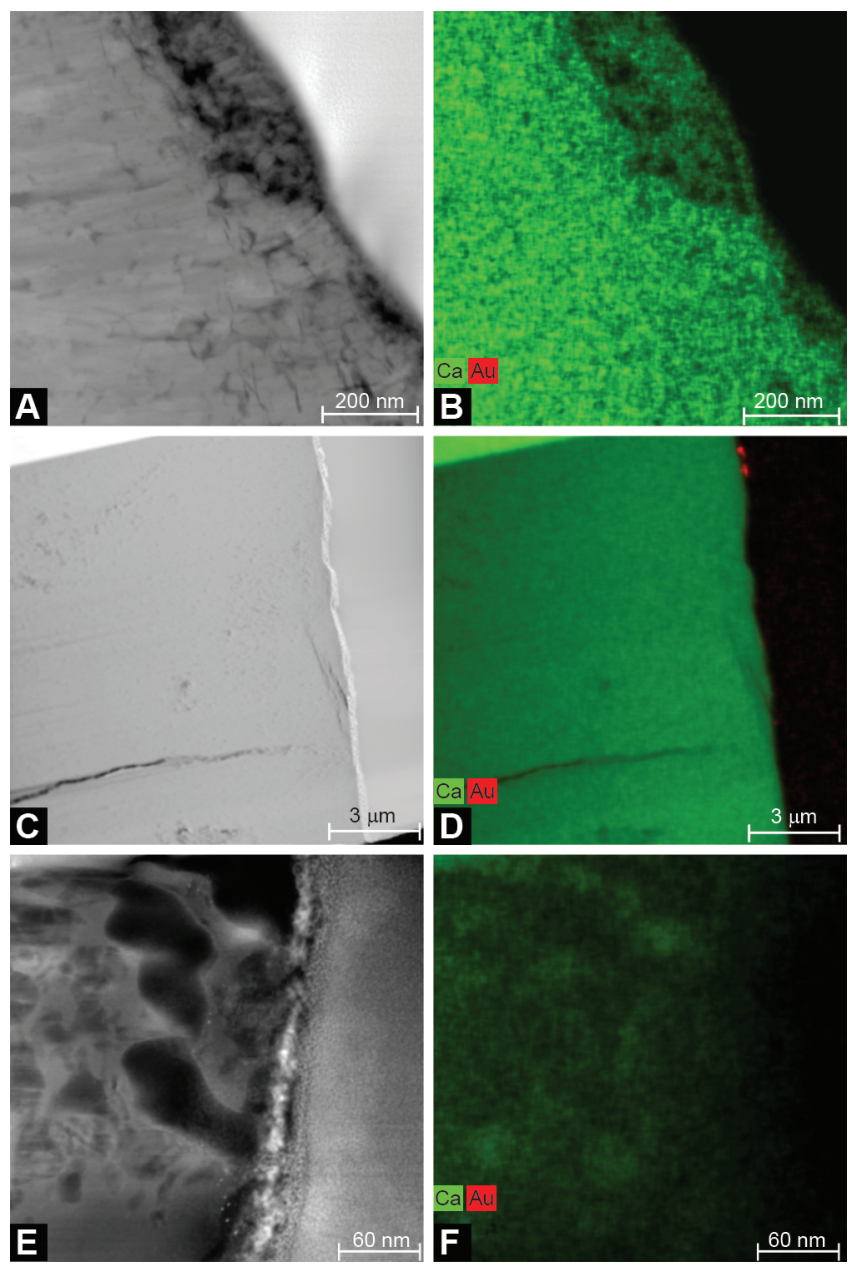

Figs $5 \mathrm{~A}$ to $\mathrm{F}$ : Scanning transmission electron microscopy (STEM) and EDS images of enamel slabs: (A) Tris buffer solution STEM image; (B) Tris buffer solution energy-dispersive X-ray spectroscopy (EDS) image; (C) Cetyltrimethylammoniumbromide-coated gold nanorods solution STEM image; (D) Cetyltrimethylammoniumbromide-coated gold nanorods solution EDS image; (E) 11-Mercaptoundecanoic acid-coated gold nanorods solution STEM image; (F) 11-Mercaptoundecanoic acid-coated gold nanorods solution EDS image

which was consistent with the micrographs obtained with CLSM. High-magnification micrographs of samples immersed in MUDA GNRs solution showed the accumulation localized to the outer enamel surface. GNRs were visible on subsurface pore areas (Fig. 5), which may be attributed to preexisting defects within the enamel substrate or cracks formed during the experimental procedure. The results of STEM imaging indicated that exposure of the enamel with chemically modified GNRs of size $\sim 20 \mathrm{~nm} \times 8 \mathrm{~nm}$ would not result in superficial penetration into the enamel.

\section{Discussion}

Novel diagnostic and therapeutic approaches using nanomaterials is a promising field in dentistry and medicine. This is because of the change in existing physicochemical properties as the size of the materials reduces to the nanometer scale. Gold nanoparticles (Au NPs) distinguish themselves from other nanoplatforms such as semiconductor quantum dots and magnetic and polymeric nanoparticles by their unique surface plasmon resonances.
These plasmonic resonance induced by photons enhances all the radiative and nonradiative properties of the nanoparticles. This offers multiple application modalities for biological and medical settings. $6,15,16$

The purpose of this study was to evaluate the potential penetration of GNRs into the tooth enamel using confocal laser scanning microscopy and scanning transmission electron microscopy. The rationale was to determine whether functional GNRs can penetrate the dental enamel creating unexpected changes in the tooth surface. Our study showed that our team was able to successfully synthesize and characterize GNRs exhibiting different surface charges. Additionally, the approach of utilizing CLSM and STEM for the evaluation of potential penetration of the nanomaterial into the tooth structure proved to be novel and feasible.

The dental enamel is the most highly mineralized and hardest tissue of the body. It is approximately $96 \%$ mineral, $3 \%$ water, and $1 \%$ organic matter by weight. The enamel hydroxyapatite is primarily composed of phosphate ions $\left(\mathrm{PO}_{4}{ }^{3-}\right)$ and calcium ions $\left(\mathrm{Ca}^{2+}\right)$. Under normal conditions, there is a stable equilibrium between the calcium and phosphate ions in saliva and the crystalline hydroxyapatite. We hypothesized that the chemical modification made to the GNRs would make a difference in the accumulation and penetration, with the anionic MUDAcoated GNRs having better affinity and penetration into the enamel. However, both qualitative CLSM and STEM images have demonstrated no difference between the two chemically modified GNRs in penetration into the enamel.

It is important to note that NPs most commonly used in food or personal care products have particle sizes that are less than $100 \mathrm{~nm} .{ }^{17}$ Despite the fact that studies are ongoing on potential toxicity of nanomaterials, there have been no studies on the ability of nanomaterials to adhere or permeate to the enamel. ${ }^{18}$ The flux into the enamel is dependent on many factors such as solubility, concentration, capillary forces, and osmotic gradients. ${ }^{19-21}$ The most critical factor seems to be the particle size. The main limitation of this study was the size of the GNRs. The synthesis of GNRs, that are as small as enamel pore sizes of approximately $2-6 \mathrm{~nm}$, could have provided better insight on size dependency. Additionally, the absence of dentinal fluid flow produced by intrapulpal pressure in vital teeth may have affected our results. With the rapid introduction of nanomaterials as a means to treat oral biofilm-related diseases, it is crucial that further studies are carried out on the potential effects of these materials on the tooth structure.

\section{Conclusion}

Within the limitations of this study, it is concluded that the use of CLSM and STEM is a feasible approach to investigate the penetration of nanomaterials into the tooth structure. Regardless of the chemical modification of the GNRs of size $\sim 20 \mathrm{~nm} \times 8 \mathrm{~nm}$, exposure of these materials did not result in superficial penetration into the enamel.

\section{ACKNOWLEDgMENTS}

This research project has been made possible through a grant from the Colgate-Palmolive Company (\#2160391). The authors thank Dong An Yang at the University of Riverside Clean Facility for helping with the SEM/STEM and Monica Romero from LLUSM AIM facility for helping with the CLSM imaging. 


\section{References}

1. Maman P, Nagpal M, Gilhotra RM, et al. Nano era of dentistry-an update. Curr Drug Deliv 2018;15(2):186-204. DOI: 10.2174/15672018 14666170825155201.

2. Freitas RA. Jr. Nanodentistry. J Am Dent Assoc 2000;131(11):1559-1565. DOI: 10.14219/jada.archive.2000.0084.

3. Uskokovic V, Bertassoni LE. Nanotechnology in dental sciences:moving towards a finer way of doing dentistry. Materials 2010;3(3):1674-1691. DOI: 10.3390/ma3031674.

4. Bhardwaj A, Bhardwaj A, Misuriya A, et al. Nanotechnology in dentistry: present and future. J Int Oral Health 2017;6(1): $121-126$.

5. Huang X, El-Sayed M. Gold nanoparticles: optical properties and implementations in cancer diagnosis and photothermal therapy. J Adv Res 2010;1(1):13-28. DOI: 10.1038/ncomms1042.

6. Yang X, Wei Q, Shao H, et al. Multivalent aminosaccharide-based gold nanoparticles as narrow-spectrum antibiotics in vivo. ACS Appl Mater Interfaces 2019;11(8):7725-7730. DOI: 10.1021/acsami.8b19658.

7. Jiao Y, Tay FR, Niu LN, et al. Advancing antimicrobial strategies for managing oral biofilm infections. Int J Oral Sci 2019;11(3):28. DOI: 10.1038/s41368-019-0062-1.

8. Mira A, Simon-Soro A, Curtis MA. Role of microbial communities in the pathogenesis of periodontal diseases and caries. J Clin Periodontol 2017;44(Suppl 18):S23-S38. DOI: 10.1111/jcpe.12671.

9. Peters MC. Strategies for noninvasive demineralized tissue repair. Dent Clin N Am 2010;54(3):507-525. DOI: 10.1016/j.cden.2010. 03.005 .

10. Hegedus C, Bistey T, Flora-Nagy E, et al. An atomic force microscopy study on the effect of bleaching agents on enamel surface. J Dent 1999;27(7):509-515. DOI: 10.1016/s0300-5712(99)00006-8.

11. Gan HY, Sousa FB, Carlo HL, et al. Enhanced transport of materials into enamel nanopores via electrokinetic flow. J Dent Res 2015;94(4): 615-621. DOI: 10.1177/0022034515572189.
12. Scarabelli L, Sanchez-Iglesias A, Perez-Juste J, et al. "Tips and Tricks" practical guide to the synthesis of gold nanorods. J Phys Chem Lett 2015;6(21):4270-4279. DOI: 10.1021/acs.jpclett.5b02123.

13. Sweeney SF, Woehrle GH, Hutchison JE. Rapid purification and size separation of gold nanoparticles via diafiltration. J Am Chem Soc 2006;128(10):3190-3197. DOI: 10.1021/ja0558241.

14. Orendorff CJ, Murphy CJ. Quantitation of metal content in the silver-Assisted growth of gold nanorods. J Phys Chem 2006;110(9): 3990-3994. DOI: 10.1021/jp0570972.

15. Kirui DK, Weber G, Talackine J, et al. Targeted laser therapy synergistically enhances efficacy of antibiotics against multidrug resistant staphylococcus aureus and pseudomonas aeruginosa biofilms. Nanomedicine 2019;20:102018. DOI: 10.1016/j. nano.2019.102018.

16. Mou XZ, Chen XY, Wang J, et al. Bacteria-instructed click chemistry between functionalized gold nanoparticles for point-of-care microbial detection. ACS Appl Mater Interfaces 2019;11(26):23093-23101. DOI: 10.1021/acsami.9b09279.

17. Weir $A$, Westerhoff $P$, Fabricius $L$, et al. Titanium dioxide nanoparticles in food and personal care products. Environ Sci Technol 2012;46(4):2242-2250. DOI: 10.1021/es204168d.

18. Besinis A, De Peralta T, Tredwin CJ, et al. Review of nanomaterials in dentistry: interactions with the oral microenvironment, clinical applications, hazards, and benefits. ACS Nano 2015;9(3):2255-2289. DOI: 10.1021/nn505015e.

19. Pashley DH, Livingston MJ, Whitford GM. The effect of molecular size on reflection coefficients in human dentine. Arch Oral Biol 1979;24(6):455-460. DOI: 10.1016/0003-9969(79)90008-6.

20. Oliver CM, Abbott PV. Entrapped air and its effects on dye penetration of voids. Endod Dent Traumatol 1991;7(3):135-138. DOI: 10.1111/j.16009657.1991.tb00198.x.

21. Vasiliadis L, Darling Al, Lever BG. The histology of sclerotic human root dentine. Arch Oral Biol 1983;28(8):693-700. DOI: 10.1016/00039969(83)90103-6. 